Check for updates

Cite this: RSC Adv., 2018, 8, 8455

Received 11th January 2018

Accepted 16th February 2018

DOI: $10.1039 / \mathrm{c} 8 \mathrm{ra00296g}$

rsc.li/rsc-advances

\section{Linc00472 suppresses breast cancer progression and enhances doxorubicin sensitivity through regulation of miR-141 and programmed cell death 4}

\author{
Pengwei Lu, Xue Yang, Yunqing Yang, Fang Wang, Lin Li and Yuanting Gu (D)*
}

The existence of drug resistance strikingly hampers the therapy of many malignancies, including breast cancer. Long non-coding RNAs (LncRNAs) have been reported to participate in the regulation of various biological processes associated with cancer progression. Whereas, the role of linc00472 in breast cancer pathogenesis and doxorubicin (ADR) resistance have not been well elucidated. In the present study, it is found that linc00472 expression was decreased in breast cancer tissues and cells. Moreover, higher linc00472 expression was positively associated with favorable disease status and prognosis for breast cancer patients. Functional analyses revealed that linc00472 overexpression suppressed proliferation and invasion, facilitated apoptosis and enhanced ADR sensitivity in breast cancer cells. Mechanistic studies discovered that linc00472 acted as a competing endogenous RNA (ceRNA) of miR-141 to sequester miR-141 from its target mRNA PDCD4 (programmed cell death 4). Furthermore, the inhibition effect of linc00472 on breast cancer cell progression and ADR resistance could be partly abrogated by miR-141 up-regulation or PDCD4 knockdown. In vivo assays also demonstrated that linc00472 hindered tumor growth by suppressing miR-141 expression and enhancing PDCD4 expression. In conclusion, linc00472 blocked breast cancer progression and induced ADR sensitivity through regulation of miR-141 and PDCD4, highlighting a potential therapeutic strategy for breast cancer patients.

\section{Introduction}

Breast cancer is the most common malignancy among women, accounting for $29 \%$ of all new diagnosed cancers. Although much progress has been made in early detection and adjuvant treatment, breast cancer is still the second major cause of cancer-triggered death in women. ${ }^{1}$ Among breast cancer patients with surgery and radio-chemotherapy treatment, approximately $30-75 \%$ breast cancer will evolve into metastatic or recurrent disease. ${ }^{2,3}$ Moreover, most patients with metastatic breast cancer were incurable with a 5 year survival rate of 20$40 \%{ }^{4}$ Doxorubicin (ADR), also named as adriamycin, is a chemotherapy regimens widely used for the treatment of metastatic breast cancer patients. ${ }^{5}$ However, the existence of chemoresistance strikingly limited the curative effect of drugs including ADR in breast cancer. ${ }^{6,7}$ Hence, it is of great importance to gain insight into the molecular mechanisms underlying ADR resistance involved in breast cancer progression in order to figure out more effective treatment strategies for breast cancer.

Department of Breast Surgery, The First Affiliated Hospital of Zhengzhou University, No. 1 Jianshe East Road, Erqi District, Zhengzhou, 450000, China. E-mail: guyuantinggyt@163.com; Tel: +86-0371-67967171
Long non-coding RNAs (lncRNAs), a class of transcripts longer than 200 nucleotides without protein coding potential, have been demonstrated to be critical mediators in various biology processes implicated in carcinogenesis and drug sensitivity/resistance. ${ }^{8}$ Also, a broad spectrum of lncRNAs are involved in breast cancer progression via modulating cell growth, apoptosis, migration and invasiveness at epigenetic, transcriptional and post-transcriptional levels. ${ }^{9}$ For instance, IncRNA NEAT1 contributed to cell growth and invasion via regulating miR-211/HMGA2 axis in breast cancer. ${ }^{10}$ Upregulation of IncRNA HOTAIR promoted cell proliferation and conferred tamoxifen resistance in breast cancer. ${ }^{11}$ IncRNA-ATB overexpression induced epithelial-mesenchymal transition (EMT) and enhanced trastuzumab resistance in breast cancer through miR-200c-ZEB1/ZNF217 pathway. ${ }^{12}$ Linc00472, located on chromosome 6q13, has been discovered to be positively correlated with favorable disease status and prognostic value together with a better chemotherapy response in breast cancer. $^{\mathbf{1 3 , 1 4}}$ Moreover, high expression of linc00472 inhibited breast cancer progression such as proliferation and migration. ${ }^{\mathbf{1 3}}$ However, the molecular mechanisms of linc00472 underlying breast cancer progression and ADR resistance have not been well elucidated till now. 
In the present study, we firstly demonstrated that linc00472 expression was down-regulated in breast cancer tissues and cells, and linc00472 expression was positively related with favorable disease status and better prognosis of breast cancer patients. Function analysis manifested that linc00472 suppressed proliferation and invasion, enhanced ADR susceptibility, and facilitated apoptosis in breast cancer cells. Further mechanism study demonstrated that linc00472 exerted its antitumor effect by regulating miR-141/PDCD4 (programmed cell death 4) axis in breast cancer.

\section{Materials and methods}

\subsection{Tissues specimens and cell culture}

Breast cancer tissues specimens $(n=40)$ and adjacent normal tissues were obtained from the breast cancer patients suffering surgical resection or biopsy between 2014 and 2016 at our hospital. Our study got the approval of Research Ethics Committee of the First Affiliated Hospital of Zhengzhou University and every patient signed the informed consent before enrolling in the study. The TNM stage of breast cancer patients was determined according to the standard of the American Joint Committee on Cancer (AJCC). All cell lines were purchased from American Tissue Culture Collection (ATCC, Manassas, VA, USA). Human mammary epithelial cell line (MCF-10A) were grown in Clonetics MEGM ${ }^{\mathrm{TM}}$ mammary epithelial cells growth medium (Lonza Walkersville, Inc. Walkersville, MD, USA) containing 5\% horse serum, cholera toxin (100 $\left.\mathrm{ng} \mathrm{ml}^{-1}\right)$, hydrocortisone (0.5 $\mu \mathrm{g} \mathrm{ml}^{-1}$ ), hEGF $\left(20 \mathrm{ng} \mathrm{ml}^{-1}\right)$ and insulin $\left(10 \mu \mathrm{g} \mathrm{ml}^{-1}\right)$. Human breast cancer cell line (MDA-MB-231, MDA-MB-453 and MCF-7) were cultured in RPMI 1640 medium (Invitrogen, Carlsbad, CA, USA) supplemented with 10\% FBS (Invitrogen). Doxorubicin (ADR) was purchased from Sigma-Aldrich (St. Louis, MO, USA). All cells were maintained in humidified incubator containing $5 \% \mathrm{CO}_{2}$ at $37{ }^{\circ} \mathrm{C}$.

\subsection{Transfection}

The full length sequences of linc00472 were amplified by PCR and constructed into pcDNA3.1 vector (Invitrogen) to form pcDNAlinc00472 overexpression plasmid. Then the putative binding sites in the pcDNA-linc00472 were mutant to generate pcDNAlinc00472-MUT plasmid. miR-141 mimic and its scramble control (miR-con) along with miR-141 inhibitor (anti-miR-141) and its scramble control (anti-miR-con) were purchased from Sigma-Aldrich (St. Louis). Linc00472 small interference RNA (silinc00472) and its scramble control (si-con) together with the siRNA of PDCD4 (si-PDCD4) and its scramble control (si-con) were synthesized by GenePharma Co., Ltd (Shanghai, China). Then, $100 \mathrm{pmol}$ oligonucleotides or $3 \mu \mathrm{g}$ plasmids were transfected into cells using Lipofectamine 2000 reagent (Invitrogen) referring to the manufacturer's instructions. The amplification primers and endonucleases were listed in Table 1.

\subsection{RT-qPCR assays}

Total RNA was isolated from breast tissues specimens or cells employing TRIzol Reagent (Invitrogen). Equal amounts of RNA was reversely transcribed into the first-strand cDNA using MMLV reverse transcriptase (Invitrogen). Then SYBR Green Realtime PCR Master Mix (TOYOBO, Osaka, Japan) was used to detect the expression patterns of linc00472, miR-141 and PDCD4 mRNA. The reverse transcription (RT) primer of miR141 and quantification primers of linc00472, miR-141, $\beta$-actin, U6 snRNA and PDCD4 were listed in Table 1 . The relative gene expression was determined by $2^{-\Delta \Delta \mathrm{Ct}}$ method with U6 snRNA as an internal control of miR-141 and $\beta$-actin as an endogenous control of linc00472 and PDCD4.

\subsection{Western blot assays}

Total protein was extracted from tissues and cells using pre-cold RIPA buffer (Thermo Scientific, Rockford, IL, USA). Then equal amounts of protein was separated on SDS-PAGE gel and transferred onto PVDF membranes (Millipore Corp. Bedford, MA, USA). Next, the membranes were blocked by $5 \%$ non-fat milk, followed by the incubation with primary antibodies against PDCD4 (sc-376430, mouse monoclonal antibody, 1 : 1000), ki-67 (sc-23900, mouse monoclonal antibody, $1: 1000$ ), $\beta$-actin (sc58673, mouse monoclonal antibody, $1: 1000$ ), activated caspase 3 (sc-22171, goat polyclonal antibody, $1: 1000$ ), procaspase 3 (sc-271759, mouse monoclonal antibody, $1: 1000$ ), GAPDH (sc-47724, mouse monoclonal antibody, $1: 1000$ ) overnight at $4{ }^{\circ} \mathrm{C}$. All antibodies were purchased from Santa Cruz Biotechnology, Inc. (Santa Cruz, CA. USA). Subsequently, the membranes were treated with HRP-conjugated secondary antibody (goat-anti-mouse polyclonal secondary antibody, ab6789, $1: 5000$; or rabbit-anti-goat polyclonal secondary antibody, ab6741, 1 : 10 000, abcam, Cambridge, UK) for $1 \mathrm{~h}$ at room temperature. At last, specific protein signal was detected employing the BeyoECL Plus agent (Beyotime, Shanghai, China).

\subsection{Luciferase reporter assays}

The partial sequences of linc00472 and PDCD4 containing putative or mutant miR-141 binding sites were amplified by PCR and cloned into pmirGLO Dual-Luciferase miRNA Target Expression Vector (Promega, Madison, WI, USA), to generate LINC00472-WT, LINC00472-MUT, PDCD4-WT (WT), PDCD4MUT (MUT). Then the constructed luciferase vectors were respectively transfected into breast cancer cells together with miRNAs (miR-con, miR-141), miRNA inhibitors (anti-miR-con, anti-miR-141), overexpression plasmids (pcDNA3.1 vector, pcDNA-Inc00472) or siRNAs (si-con, si-lnc00472). At $48 \mathrm{~h}$ after transfection, the luciferase activity was determined using DualLuciferase Reporter Assay System (Promega) following the manufacturer's instructions.

\subsection{EdU (5-ethynyl-2'-deoxyuridine) immunofluorescence and flow cytometry assays}

Cell proliferation patterns were also measured by EdU (5ethynyl-2'-deoxyuridine) immunofluorescence assays using Cell-Light TM EdU kit (RiboBio) referring the manufacturer's instructions. For immunofluorescence assays, transfected cells $\left(1 \times 10^{5}\right.$ per well, 96-well plate) were labeled with complete 
Table 1 Primers used in this study

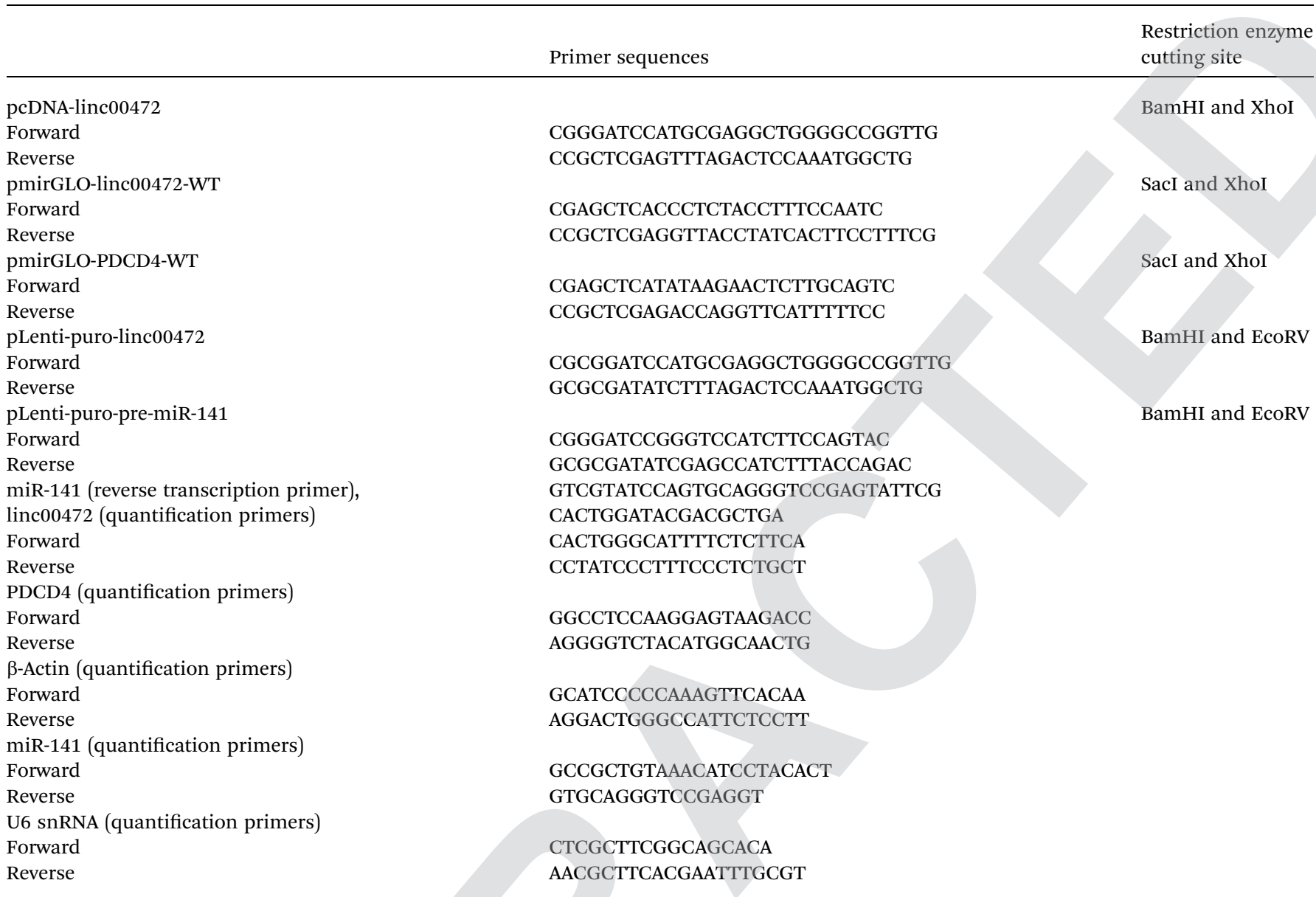

medium containing $50 \mu \mathrm{M}$ EdU for $2 \mathrm{~h}$, and then fixed using $4 \%$ paraformaldehyde for $30 \mathrm{~min}$ at room temperature, followed by the addition of $2 \mathrm{mg} \mathrm{ml}^{-1}$ glycine for $5 \mathrm{~min}$. Next, $100 \mu \mathrm{l} 1 \times$ Apollo solution was added into each well. After incubation for $30 \mathrm{~min}$ in dark, Apollo solution was discarded and $100 \mu \mathrm{l}$ of $0.5 \%$ TritonX-100 was added into each well for $10 \mathrm{~min}$. After 3 times of repetition, cells were stained with DAPI solution $(1 \mu \mathrm{g}$ $\mathrm{ml}^{-1}$, Beyotime, Shanghai, China) for $20 \mathrm{~min}$, and then imaged using a confocal microscopy. For flow cytometry assays, transfected cells were labeled with complete medium containing 50 $\mu \mathrm{M}$ EdU for $2 \mathrm{~h}$, followed by the digestion with $0.25 \%$ trypsin for $1 \mathrm{~min}$ and fixation with $4 \%$ paraformaldehyde for $15 \mathrm{~min}$. Then cells were stained using Apollo and DAPI solutions as described above. At last, cells were collected at $1000 \mathrm{rpm}$ and resuspended in $300 \mu \mathrm{l}$ PBS prior to subjecting to flow cytometer (BD Biosciences, San Jose, CA, USA).

\subsection{Colony formation assay}

Briefly, transfected MCF-7 and MDA-MB-453 cells were seeded in 6-well plates and incubated for 15 days. Next, the treated cells were fixed using acetic acid/methanol $1: 7(\mathrm{v} / \mathrm{v})$ and stained with $0.5 \%$ crystal violet solution (Sigma-Aldrich). As last, cell colonies were counted under an inverted microscope.

\subsection{Cell cycle and apoptosis analyses}

At $48 \mathrm{~h}$ after transfection, MCF-7 and MDA-MB-453 cells were treated for further analyses of cell cycle and apoptosis. For cell cycle analysis, cells were fixed using $80 \%$ ethanol at $-20{ }^{\circ} \mathrm{C}$ overnight and then treated with DNase-free RNase A (Thermo Scientific, $50 \mu \mathrm{g} \mathrm{ml}^{-1}$ ) and propidium iodide (PI, Sigma-Aldrich, $50 \mu \mathrm{g} \mathrm{ml}^{-1}$ ) for $1 \mathrm{~h}$ at room temperature in the dark, followed by the analysis on flow cytometer (BD Biosciences). Cell apoptosis rate was determined by Annexin V-FITC/PI Apoptosis Detection kit (Beyotime) referring to the instructions of manufacturer.

\subsection{Cell viability assays and $\mathrm{IC}_{50}$ value determination}

Cell Proliferation Kit I (MTT) (Sigma-Aldrich) was used to assess cell viability and $\mathrm{IC}_{50}$ value (defined as the half maximal inhibitory concentration of drugs) of ADR. At the indicated time points $(0,24,48,72 \mathrm{~h})$ after transfection, MTT assays were performed to detect cell viability. Additionally, transfected cells were inoculated into 96-well plates and incubated for $48 \mathrm{~h}$, followed by the addition of gradient concentrations of ADR $(0.001,0.01,0.1,1,10,100 \mu \mathrm{M})$ for another $24 \mathrm{~h}$ and $\mathrm{IC}_{50}$ value determination using MTT assays. MTT assays were carried out as follows. Briefly, MTT solution was added into the 96-well plates and incubated for $4 \mathrm{~h}$. Then cell medium containing MTT 
was removed and $150 \mu$ limethyl sulfoxide (DMSO) was added into each well to dissolve formed formazan crystals. At last, the absorbance was measured at the wavelength of $570 \mathrm{~nm}$.

\subsection{RNA immunoprecipitation (RIP) assays}

RIP assays were carried out employing the EZ-Magna RIP kit (Millipore, Billerica, MA, USA) according to the manufacturer's protocol. Briefly, MCF-7 and MDA-MB-453 cells were lysed with pre-cold RIPA buffer and then incubated with magnetic beads and primary antibody against Ago2 (Millipore) or IgG. Then the complex was treated with RNase-free DNase I (Promega, 10U) for $30 \mathrm{~min}$ at $37^{\circ} \mathrm{C}$ and digested with proteinase $\mathrm{K}\left(1 \mathrm{mg} \mathrm{ml}{ }^{-1}\right)$ for $20 \mathrm{~min}$ at $45^{\circ} \mathrm{C}$ to remove the superfluous DNA and protein. Finally, RT-qPCR assays were used to detect the expression patterns of specific RNAs in purified RNA.

\subsection{Subcellular fractionation}

Cytoplasmic and Nuclear RNA Purification Kit (Norgen, Thorold, ON, Canada) was employed to isolate the nuclear and cytoplasm fractions. Then the expression levels of GAPDH, U6 and linc00472 in nuclear and cytoplasm fractions of MCF-7 and MDA-MB-453 cells were detected using RT-qPCR assays.

\subsection{Transwell invasion assays}

Transwell assays were performed to assess cell invasion capability. Briefly, approximately $1 \times 10^{5}$ MCF-7 and MDA-MB-453 cells were inoculated into the upper chamber containing matrigel (BD Biosciences). Cells in the upper chamber were maintained in serum-free RPMI-1640 medium, while complete medium with $5 \%$ FBS was added to the lower chamber. After incubation for $48 \mathrm{~h}$, the cells adhered to lower surface were fixed in the $100 \%$ pre-cold methanol, staining with $0.1 \%$ crystal violet solution (Sigma-Aldrich) and then counted by microscope.

\subsection{Lentivirus production and infection}

pLenti-puro was a gift from Ie-Ming Shih (Addgene, Cambridge, Massachusetts, USA). The sequences of miR-141 precursor and linc00472 were amplified by PCR from genomic DNA and subcloned into pLenti-puro vector to generate the overexpression plasmid of miR-141 and linc00472, named as lentipre-miR-141 and lenti-linc00472. Then the lentiviral plasmids were respectively transfected into HEK293T cells along with psPAX2 and pMD2.G plasmids (Addgene). After transfection of $72 \mathrm{~h}$, cell medium containing lentivirus were collected and filtered using $0.45 \mathrm{~mm}$ pore filter. Then, MCF-7 cells were infected with treated lentivirus, followed by the screening with puromycin (Sigma-Aldrich) for about 1 week so as to get stable lentivirus-transfected cell lines.

\subsection{Tumor formation in nude mice}

All experiment processes were performed according to the national standard of the care and use of laboratory animals and got the approval of Institutional Committee for Animal Research. BALB/c athymic nude mice were obtained from Hubei Research Center of Laboratory Animal (Wuhan, China).
Approximately $5 \times 10^{6}$ MCF-7 cells stably transfected with lentipre-miR-141, lenti-linc00472, lenti-linc00472+lenti-pre-miR-141 were subcutaneously injected into the armpit of nude mice. At 7 days post-inoculation, tumor volumes were detected using a caliper once a week for 6 weeks. Tumors were photographed and tumor weights were measured at 42 days after injection. Also the expression levels of miR-141, linc00472 and ki-67 in tumors tissues were analyzed by RT-qPCR and western blot assays.

\subsection{Statistical analysis}

All assays were repeated at least three times with the results displayed as mean \pm standard deviation (S.D.). Overall survival (OS) was calculated from the first surgery until death or the last follow-up investigation and was analyzed by Kaplan-Meier analysis. The correlation studies were performed using Spearman's correlation analysis. Student's $t$-test and one-way ANOVA were used to compare the significant difference of different groups. $\mathrm{IC}_{50}$ value was measured through SPSS software employing probit regression analysis. $P<0.05$ meant the difference was statistically significant.

\section{Results}

3.1. Linc00472 expression was down-regulated in breast cancer tissues and cells

Firstly, RT-qPCR assays were performed to detect the expression pattern of linc00472 in breast cancer tumor tissues. As shown in Fig. 1A, linc00472 expression level was remarkably decreased in 40 pairs of breast cancer tissues (Cancer) compared with adjacent normal tissues (Normal) (Fig. 1A). We also found that linc00472 level was notably down-regulated with the development of lymph-node metastasis in breast cancer (Fig. 1B). In addition, low-level expression of linc00472 might be related with distant metastasis of breast cancer (Fig. 1C). Subsequently, we measured linc00472 expression in several breast cancer cell lines (MDA-MB-231, MDA-MB-453, MCF-7). The results showed that linc00472 expression was markedly reduced in breast cancer cell lines compared with human normal mammary epithelial cell line MCF-10A (Fig. 1D). Kaplan-Meier survival analysis indicated that patients with low linc00472 expression displayed a poorer overall survival when compared to that with high linc00472 expression ( $P=0.0014$, log-rank test) (Fig. 1E). All these data indicated that dysregulation of linc00472 might be associated with the development and prognosis of breast cancer.

\subsection{Ectopic expression of linc00472 suppressed proliferation and invasion of breast cancer cells}

To delineate the role of linc00472 in breast cancer progression, pcDNA-linc00472 overexpression plasmid (linc00472) was constructed and transfected into breast cancer cells (MCF-7 and MDA-MB-453). RT-qPCR results showed that transfection of pcDNA-linc00472 strikingly enhanced linc00472 expression in MCF-7 and MDA-MB-453 cells compared with empty vector (Fig. 2A), indicating that pcDNA-linc00472 could be used for the 
A

following gain-of-function experiments. Then we proceeded to explore the effect of linc00472 on proliferation of breast cancer cells. MTT assays manifested that enforced expression of linc00472 markedly suppressed proliferation of MCF-7 and MDA-MB-453 cells (Fig. 2B and C). EdU assays also showed that overexpression of linc00472 greatly decreased the number of EdU-positive cells compared with control vector (Fig. 2D and E). Moreover, colony formation assays disclosed that the number of formed colonies was notably lower in linc00472-overexpressing group than that in control vector group (Fig. 2F). Also, cell cycle distribution exhibited that cell proportion in G0/G1 phase was increased and cell percentage in S-phase population was reduced in MCF-7 and MDA-MB-453 cells transfected with linc00472 compared with mock cells (Fig. 2G and H). All these data revealed that linc00472 up-regulation hampered proliferation capability of breast cancer cells. Then, we went on to explore the effect of linc00472 on invasion of breast cancer cells. As presented in Fig. 2I and J, transwell invasion assays revealed that the invasion ability of MCF-7 and MDA-MB-453 cells was weakened after introduction of linc00472-overexpression plasmid. Taken together, these data suggested that linc00472 overexpression blocked proliferation and invasion of breast cancer cells.

\subsection{Linc00472 overexpression improved ADR} chemosensitivity and enhanced ADR-mediated apoptosis in breast cancer cells

Then we proceeded to investigate the effect of linc00472 on ADR chemoresistance in breast cancer cells. To determine half maximal inhibitory concentration $\left(\mathrm{IC}_{50}\right)$ of $\mathrm{ADR}$ in breast cancer cells, MCF-7 cells and MDA-MB-453 cells transfected with pcDNA3.1 vector or pcDNA-linc00472 were treated with different concentration of ADR $(0.001,0.01,0.1,1,10,100 \mu \mathrm{M})$ for $48 \mathrm{~h}$. Then, MTT assays were performed to determine cell survival rate and $\mathrm{IC}_{50}$ values of ADR. As shown in Fig. $3 \mathrm{~A}$ and $\mathrm{B}$, introduction of linc00472 resulted in a significant reduction of breast cancer cell viability compared with empty vector at ADR treatment concentration ranging from $0.001 \mu \mathrm{M}$ to $10 \mu \mathrm{M}$. Moreover, we demonstrated that the $\mathrm{IC}_{50}$ values of $\mathrm{ADR}$ were 
A

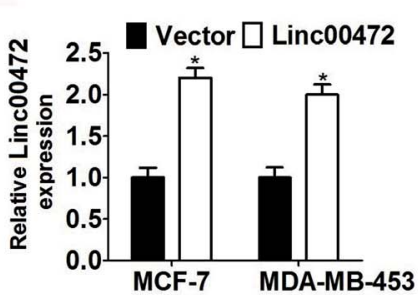

D

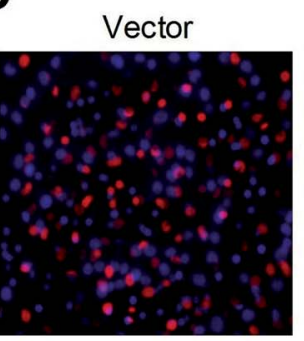

MCF-7
B

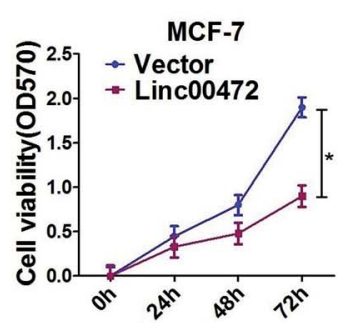

C

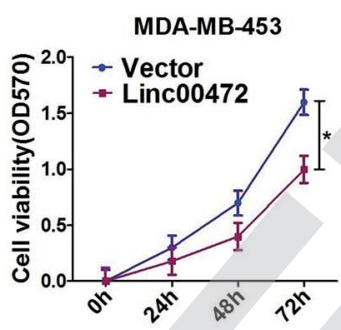

E

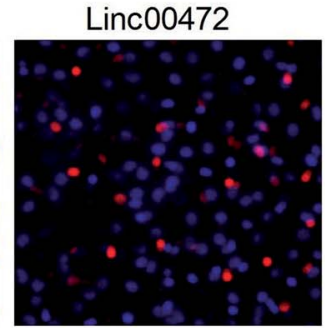

7
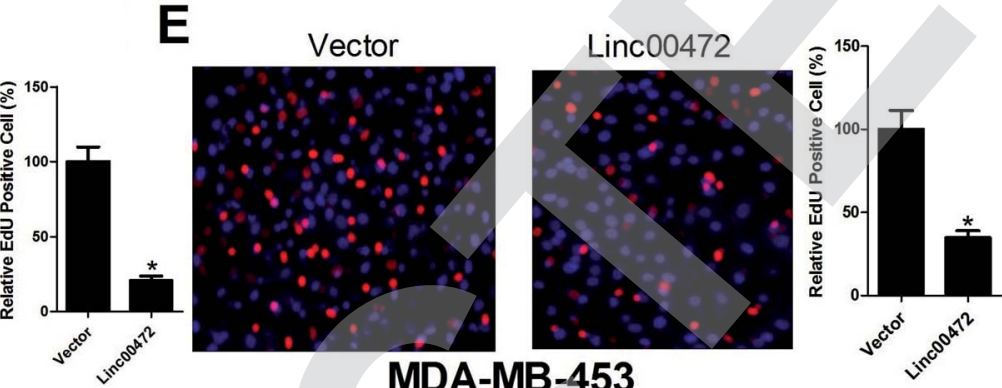

MDA-MB-453

$\mathbf{F}$

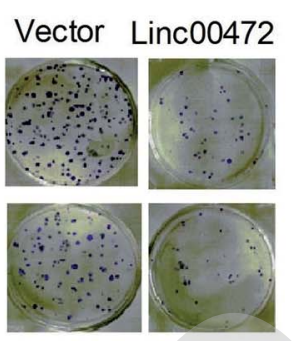

G
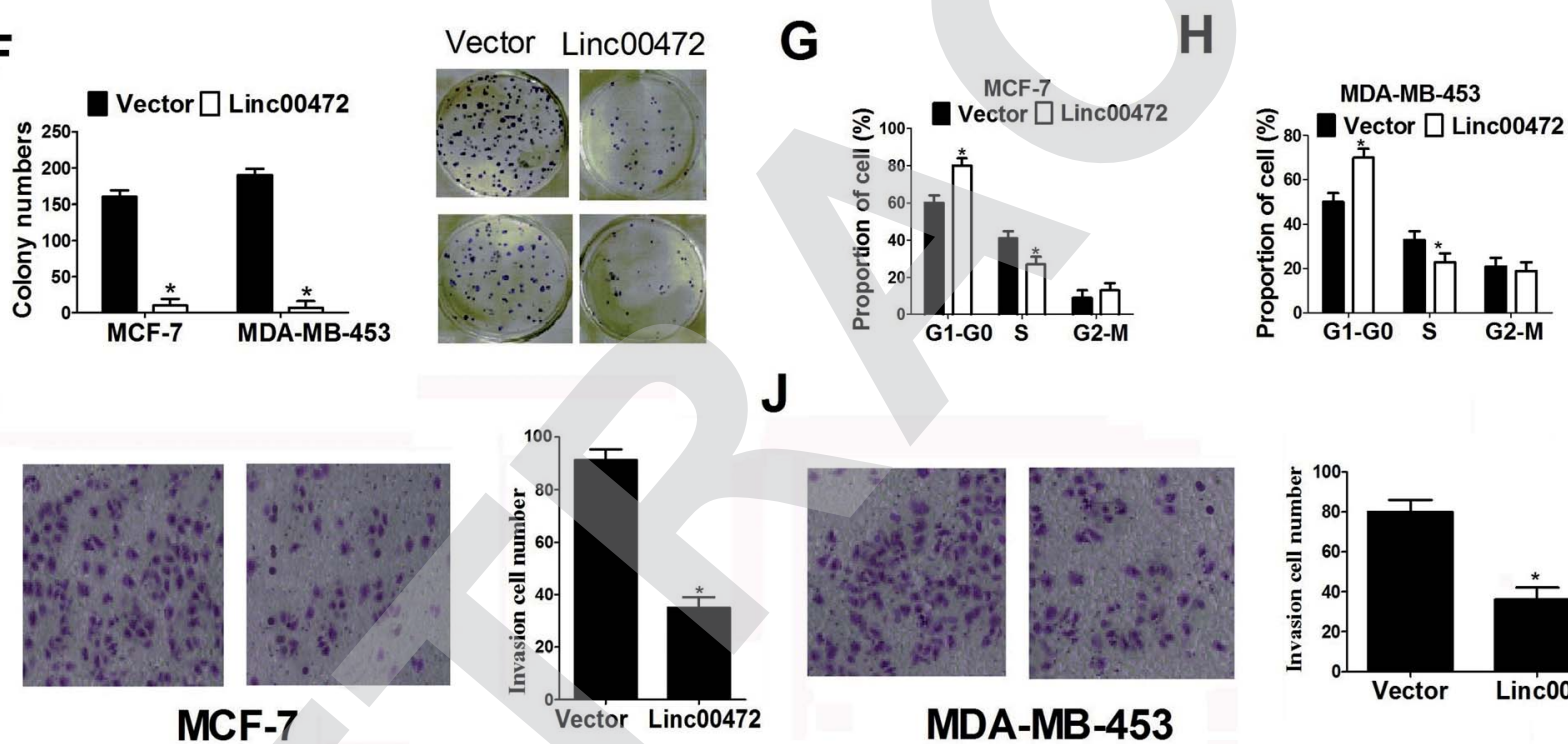

J

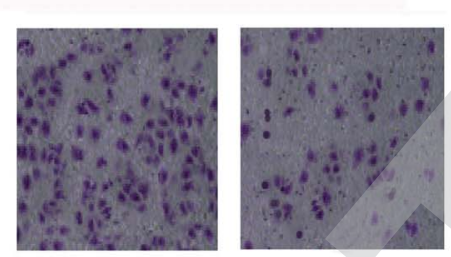

MCF-7
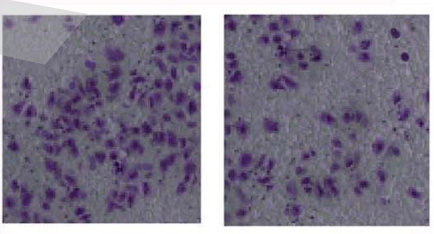

MDA-MB-453

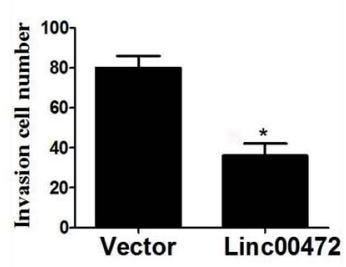

Fig. 2 Linc00472 overexpression suppressed breast cancer cells proliferation and invasion. MCF-7 and MDA-MB-453 cells were transfected with pcDNA3.1 or pcDNA-linc00472 vector. (A) RT-qPCR assay was performed to measure linc00472 expression at $48 \mathrm{~h}$ after transfection. (B and C) MTT assay was used to evaluate cell viability at the indicated time points $(0,24,48,72 \mathrm{~h})$ posttransfection. (D and E) EdU-positive cells percentage was detected by EdU immunofluorescence staining and flow cytometric analysis at $48 \mathrm{~h}$ after transfection. (F) Transfected cells were seeded in 6 well plates and incubated for 15 days, then cell colony number was assessed. ( $G$ and $H$ ) Cell cycle distribution was determined by flow cytometry $48 \mathrm{~h}$ posttransfection. ( and $\mathrm{J}$ ) Cell invasion capability was monitored using transwell invasion assays at $48 \mathrm{~h}$ after transfection. $* P<0.05$.

considerably decreased in linc00472-treated MCF-7 and MDAMB-453 cells compared with control cells (vector). In a word, up-regulation of linc00472 expression increased ADR chemosensitivity of breast cancer cells. Next, to assess whether linc00472-induced sensitivity to ADR was associated with apoptosis, MCF-7 cells and MDA-MB- 453 cells transfected with pcDNA3.1 vector or pcDNA-linc00472 were treated with $0.1 \mu \mathrm{M}$ ADR for $48 \mathrm{~h}$. Flow cytometry analysis demonstrated that linc00472 overexpression enhanced ADR-induced apoptosis in MCF-7 cells and MDA-MB-453 (Fig. 3C and D). Moreover, western blot assays revealed that the level of activated caspase 3 was markedly elevated in linc00472-overexpressing MCF-7 and MDA-MB-453 cells compared with control cells (vector) (Fig. 3E and F). In a word, these results revealed that linc00472 overexpression enhanced ADR chemosensitivity and promoted ADRmediated apoptosis in breast cancer cells.

\subsection{Linc00472 acted as a decoy of miR-141}

To further investigate the molecular mechanism of linc00472 in breast cancer progression, miRcode online website was used to search for the potential target miRNAs of linc00472. The results 
A

C

E
MCF-7
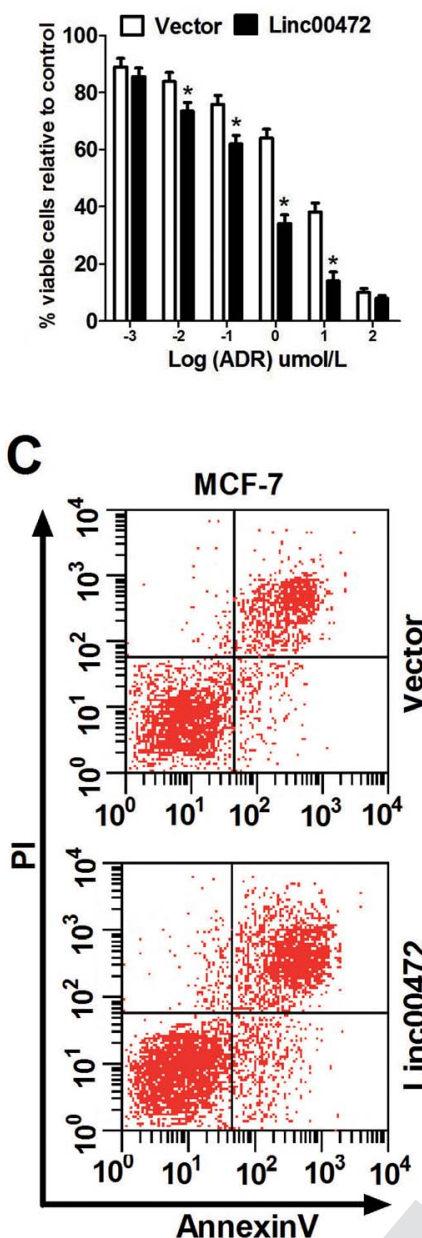

AnnexinV
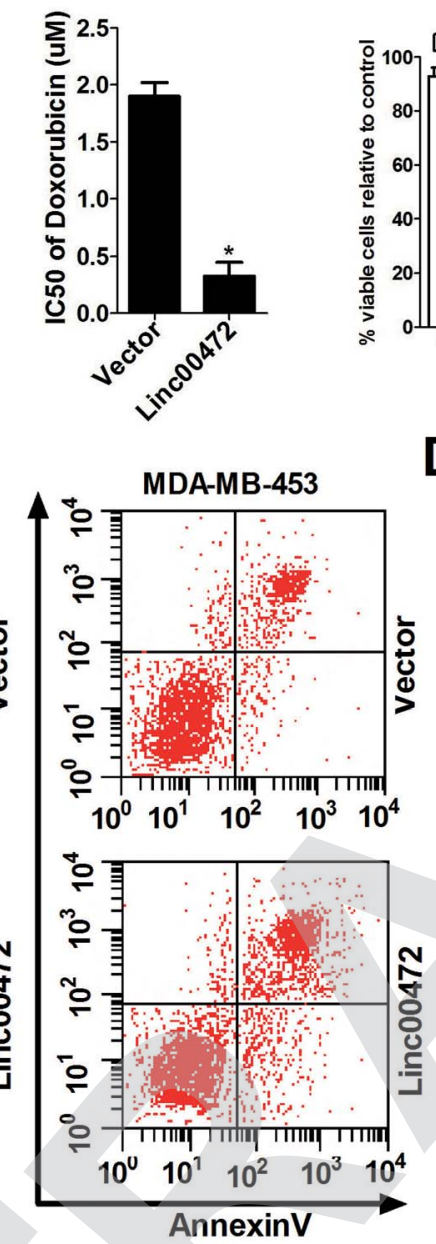

MDA-MB-453
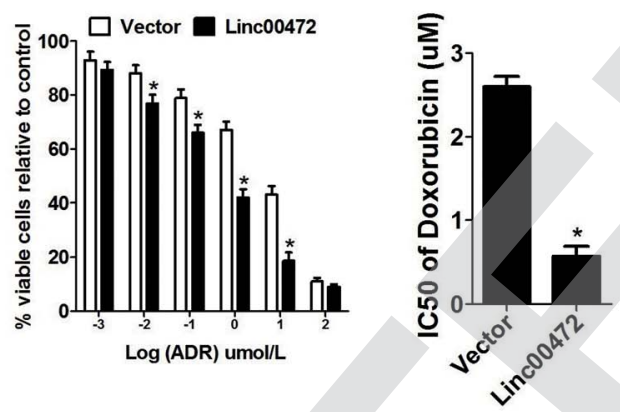

D
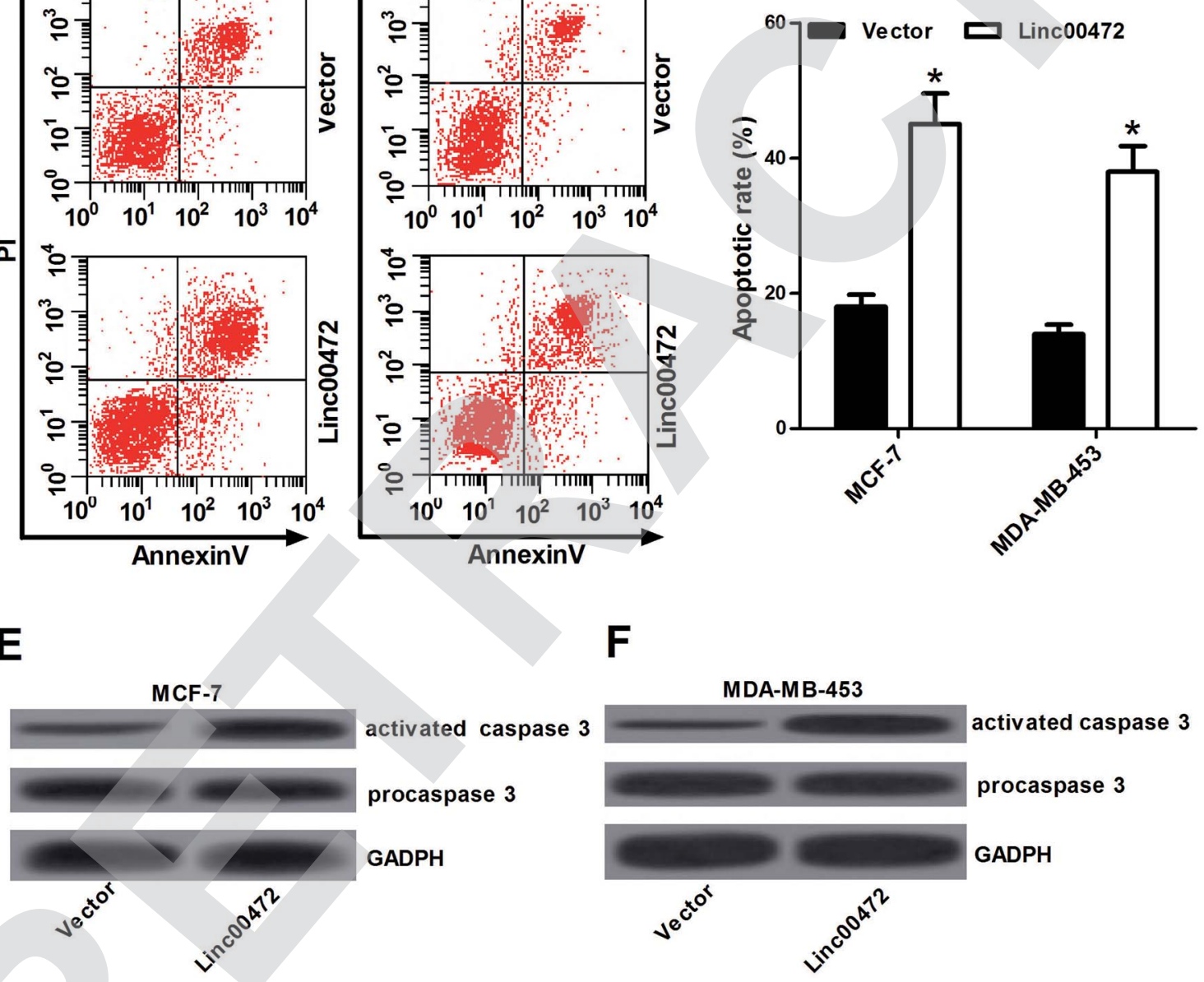

MDA-MB-453

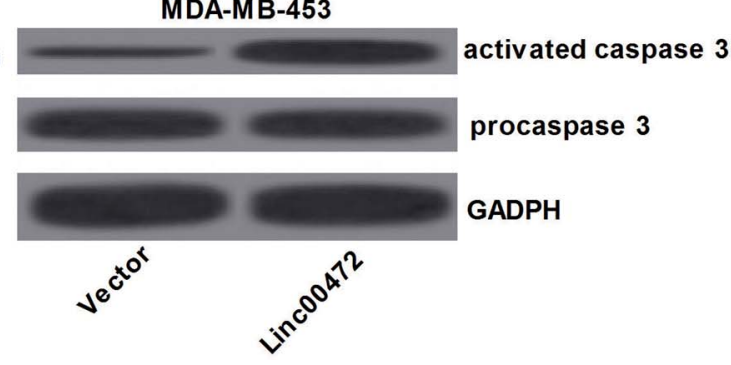

Fig. 3 Linc00472 overexpression increased doxorubicin (ADR) chemosensitivity and induced ADR-mediated apoptosis in breast cancer cells. (A and B) MCF-7 cells and MDA-MB-453 cells were transfected with pcDNA3.1 vector or pcDNA-linc00472 for 24 h, followed by treatment with different concentration of ADR $(0.001,0.01,0.1,1,10,100 \mu \mathrm{M})$ for $48 \mathrm{~h}$. Then MTT assays were performed to determine cell survival rate and IC $\mathrm{C}_{50}$ values of ADR. (C-F) MCF-7 cells and MDA-MB-453 cells transfected with pCDNA3.1 vector or pcDNA-linc00472 were treated with $0.1 \mu M$ ADR for $48 \mathrm{~h}$, followed by analysis of apoptotic rate by flow cytometry (C and D), as well as assessment of activated caspase 3 and procaspase 3 protein levels by western blot ( $\mathrm{E}$ and $\mathrm{F}) . * P<0.05$.

showed there existed some complementary sites between linc00472 and miR-141 (Fig. 4A). To explore the potential interaction between linc00472 and miR-141, cellular fractionation assays were performed to identify the subcellular location of linc00472 in MCF-7 and MDA-MB-453 cells. As displayed in
Fig. $4 \mathrm{~B}$ and $\mathrm{C}$, linc00472 was substantially enriched in the cytoplasmic fraction, indicating that linc 00472 had a chance to interact with cytoplasmic miRNAs. Next, to further confirm the direct interaction between linc00472 and miR-141, the widetype sequence of linc00472 complementary with miR-141 or 
A

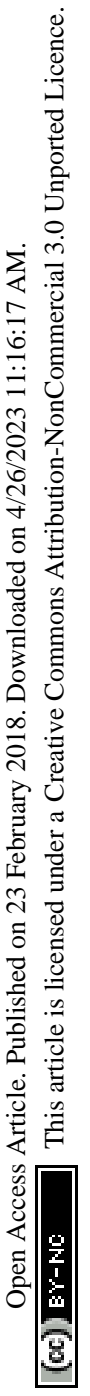

F

\section{Linc00472-WT \\ miR-141 \\ Linc00472-MUT \\ 5' UgGUACUUUCUACCAACACUGG \\ 5' UgGUACUUUCUACCCCUGACUU 3 MUT \\ 3' 5 3}

D
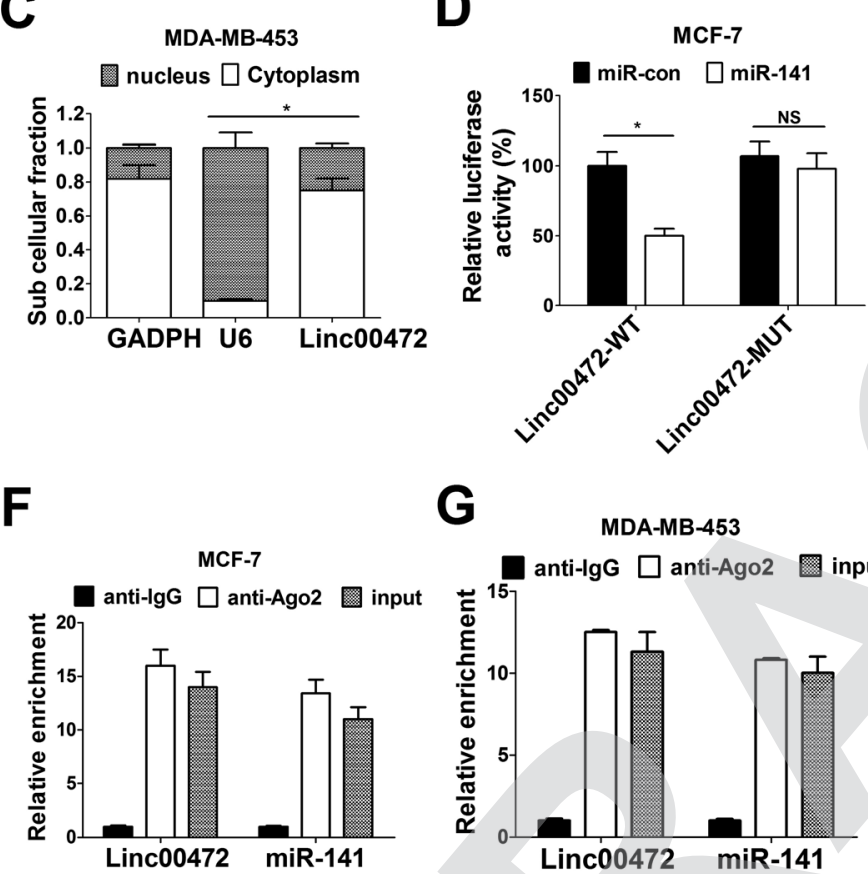

G
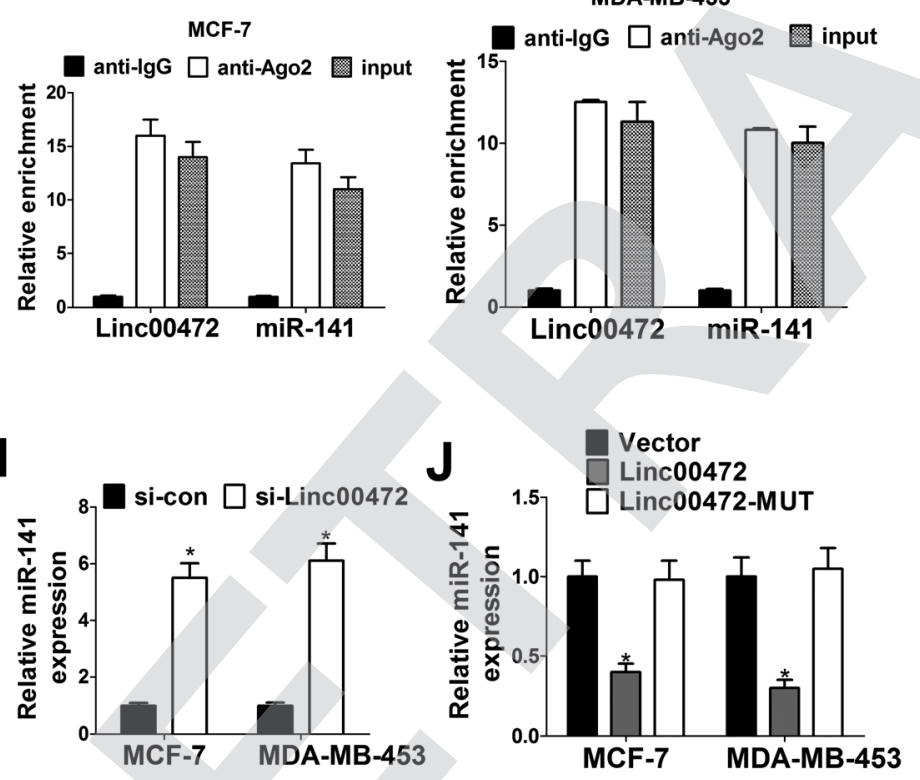

B 造

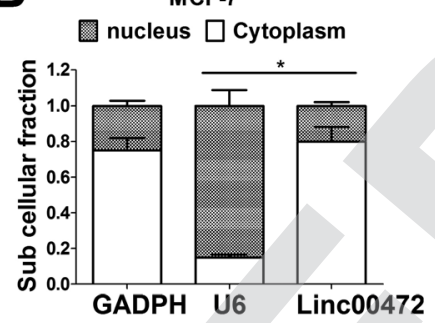

E
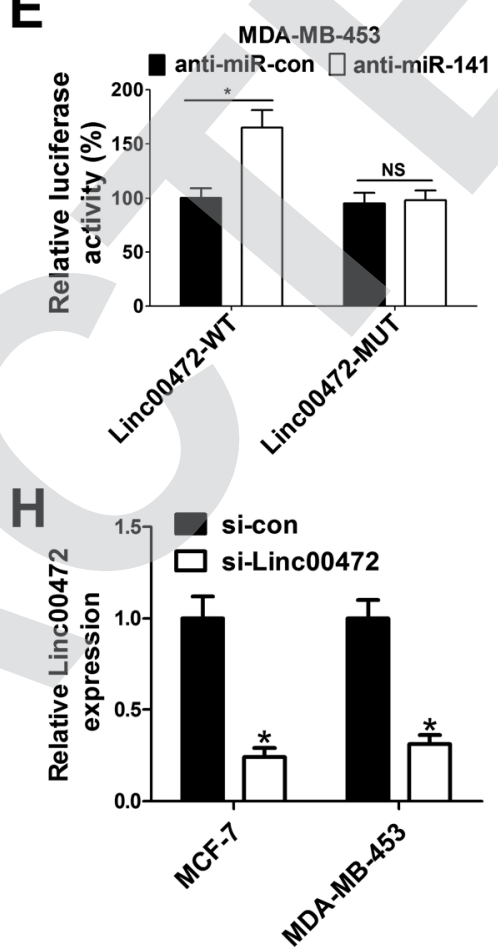

Fig. 4 Linc00472 directly inhibited miR-141 expression. (A) The predicted binding sites between linc00472 and miR-141 together with the mutant sites in the linc00472-MUT vector. (B and C) RNA of nucleus and cytoplasm in MCF-7 and MDA-MB-453 cells was isolated by subcellular fractionation assays. Then expressions of GAPDH, U6 snRNA and linc00472 were quantified by RT-qPCR assays with U6 snRNA as a nucleus control and GAPDH as a cytoplasm control. (D and E) MCF-7 cells were co-transfected with linc00472-WT or linc00472-MUT reporter and miRcon or miR-141, and MDA-MB-453 cells were co-transfected with linc00472-WT or linc00472-MUT reporter and anti-miR-con or anti-miR-141. At $48 \mathrm{~h}$ after transfection, luciferase activity was detected by Dual-Luciferase reporter assays. ( $\mathrm{F}$ and $\mathrm{G}$ ) RIP assays were performed using IgG or Ago2 antibody in MCF-7 and MDA-MB-453 cells, followed by the detection of linc00472 and miR-141 expression in immunoprecipitate. IgG performed as a negative control and input as a positive control. ( $\mathrm{H}$ and I) RT-qPCR assay was carried out to analyze the expressions linc00472 and miR-141 in MCF-7 and MDA-MB-453 cells transfected with si-linc00472 or si-con. (J) miR-141 expression was detected in MCF-7 and MDA-MB453 cells transfected with pcDNA3.1, pcDNA-linc00472 or pcDNA-linc00472-MUT vector using RT-qPCR assay. (K) Spearman's correlation analysis of linc 00472 and miR-141 expression in 40 cases of breast cancer tissues. $* P<0.05$.

its mutant sequence was subcloned into luciferase reporter vector to generate linc00472-WT or linc00472-MUT luciferase reporter. Subsequent luciferase assays revealed that the relative luciferase activity of linc00472-WT systems was markedly decreased by miR-141 overexpression, but was notably increased following miR-141 depletion (Fig. 4D and E). 
However, the upregulation or downregulation of miR-141 had no effect on luciferase activity of linc00472-MUT systems compared with their corresponding counterparts (Fig. 4D and E). RIP assays also manifested that linc00472 and miR-141 was substantially enriched by Argonaute 2 (Ago2) antibody in MCF-7 and MDA-MB-453 cells when compared to IgG group (Fig. 4F and $\mathrm{G})$. To observe the actual influence of linc00472 on miR141, si-linc00472 was transfected into MCF-7 and MDA-MB453 cells. At first, the knockdown efficiency of si-linc00472 was measured with the results showing that si-linc00472 transfection effectively decreased linc00472 expression in MCF-7 and MDA-MB-453 cells (Fig. 4H). As expected, knockdown of linc00472 gave rise to a significant increase of miR-141 expression in MCF-7 and MDA-MB-453 cells (Fig. 4I). Moreover, transfection of linc00472-overexpression plasmid containing miR-141 binding sites markedly suppressed miR-141 expression in MCF-7 and MDA-MB-453 cells, while introduction of linc00472-overexpression plasmid with mutant miR-141 binding sites (linc00472-MUT) had no effect on miR-141 level (Fig. 4J). Additionally, Spearman's correlation analysis also found that miR-141 expression level was inversely associated with linc00472 level in 40 cases of breast cancer tissues (Fig. 4K). Collectively, these results proved that linc00472 suppressed miR-141 expression by direct interaction.

\subsection{Linc00472 up-regulated PDCD4 expression by sponging miR-141}

Accumulating evidence shows that miRNA exerts function by hindering target mRNAs expression. Hence, TargetScan website was employed to search for the potential target mRNAs of miR141. Prediction results showed that there existed some complementary sites between PDCD4 and miR-141 (Fig. 5A). To further validate the interaction between linc00472, miR-141 and PDCD4, wide-type or mutant-type PDCD4-3'UTR luciferase reporter vector was constructed, followed by co-transfected with (miR-con, miR-141, miR-141+pcNDA3.1 vector, or miR141+pcNDA-linc00472) or (anti-miR-con, anti-miR-141, antimiR-141+si-con, or anti-miR-141+si-linc00472) into MCF-7 or MDA-MB-453 cells. As shown in Fig. 5B, introduction of miR141 mimic strikingly decreased luciferase activity of wide-type PDCD4-3'UTR systems, while linc00472 overexpression notably abolished this effect of miR-141. Moreover, the luciferase activity of WT-PDCD4-3'UTR systems was enhanced by miR-141 inhibitor, while this effect of anti-miR-141 was substantially abrogated by linc00472 knockdown. However, change of miR-141 or linc00472 expression had no influence on luciferase activity of mutant-type PDCD4-3'UTR systems (Fig. 5B and C). In a word, miR-141 directly interacted with PDCD4$3^{\prime}$ UTR region, while this interaction was attenuated by linc00472. Then, we continued to demonstrate the actual effect of miR-141 or linc00472 on PDCD4 expression. The results unveiled that miR-141 expression level was markedly enhanced by miR-141 mimic in MCF-7 cells, but was notably suppressed by pcDNA-linc00472 overexpression plasmid (Fig. 5D). Moreover, linc00472-induced decrease in miR-141 expression was reversed by transfection with miR-141 mimic (Fig. 5D).
Conversely, anti-miR-141 curbed miR-141 expression and knockdown of linc00472 facilitated miR-141 expression in MDA-MB-453 cells (Fig. 5E). Moreover, miR-141 inhibition attenuated si-linc00472-mediated increase on miR-141 expression (Fig. 5E). We further demonstrated that PDCD4 expression was suppressed by miR-141, but was increased by linc00472 overexpression in MCF-7 cells. In addition, miR-141 overexpression abated pcDNA-linc00472-mediated enhancement effect on PDCD4 expression (Fig. 5F). Conversely, miR-141 inhibitor contributed to PDCD4 expression and linc00472 knockdown suppressed PDCD4 expression in MDA-MB-453 cells. Also, introduction of miR-141 inhibitor abrogated silinc00472-mediated inhibition effect on PDCD4 expression (Fig. 5G). Spearman's correlation analyses revealed a positive relevance between linc00472 and PDCD4 expression (Fig. 5H), as well as an inverse correlation between miR-141 and PDCD4 (Fig. 5I) expression in 40 cases of breast cancer tissues. All these data suggested that linc00472 could sponge miR-141 to affect PDCD4 expression.

\section{6. miR-141 overexpression or PDCD4 knockdown} abolished the inhibition effect of linc00472 on breast cancer cell progression

Plentiful studies demonstrated that lncRNAs acted as ceRNAs of miRNAs to sequester them from target mRNAs and enhance mRNA expression. As mentioned above, linc00472, miR-141 and PDCD4 was closely associated with each other, hence, we proceeded to investigate their function relevance in MCF-7 cells. As displayed in Fig. 6A, overexpression of linc00472 abated the inhibition effect of miR-141 on PDCD4 expression, and upregulating miR-141 also weakened linc00472-mediated enhancement on PDCD4 expression. Moreover, we further validated that depletion of PDCD4 attenuated linc00472induced promotion effect on PDCD4 expression, and linc00472 overexpression could also abate si-PDCD4-triggered diminishment in PDCD4 expression (Fig. 6B). Function analysis revealed that the inhibitory effects of linc00472 overexpression on cell proliferation, invasion and ADR resistance were markedly abrogated by miR-141 up-regulation or PDCD4 knockdown, and the promotive effects of miR-141 overexpression or PDCD4 knockdown on cell proliferation, invasion and ADR resistance were significantly overturned following restoration of linc00472 expression (Fig. 6C-G). Collectively, these results manifested that linc00472 suppressed breast cancer progression by regulating miR-141 and PDCD4.

\subsection{Linc00472 blocked tumor growth in breast cancer in vivo}

To further investigate the effect of miR-141 and linc00472 on tumor growth, MCF-7 cells stably transfected with lenti-con (control), lenti-pre-miR-141, lenti-linc00472 or lenti-linc00472+lenti-pre-miR-141 were subcutaneously injected into the armpit of nude mice. Tumor volume was detected once every 7 days for 42 days using a caliper. Tumor weight was measured at 42 days after implantation. As presented in Fig. 7A-C, overexpression of miR-141 notably accelerated tumor growth, while 
A

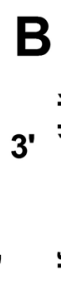

Mutant PDCD4 3'UTR 5'...GUCGgAUUACCAAACUCUGUAUU ...3'

PDCD4 3'UTR 5'... GUCGGAUUACCAAAACACUGCCYU... 3'
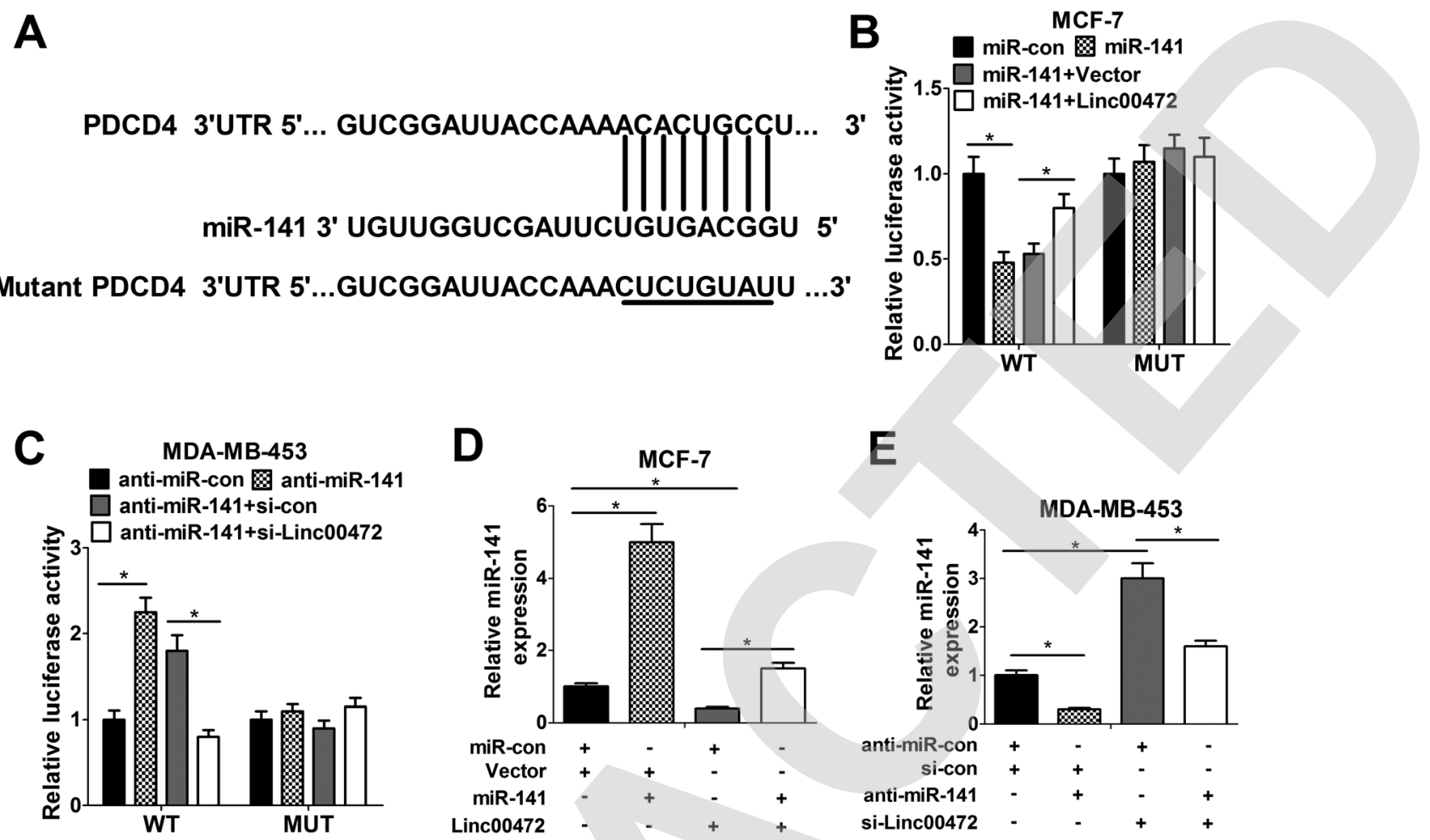

$\mathbf{F}$
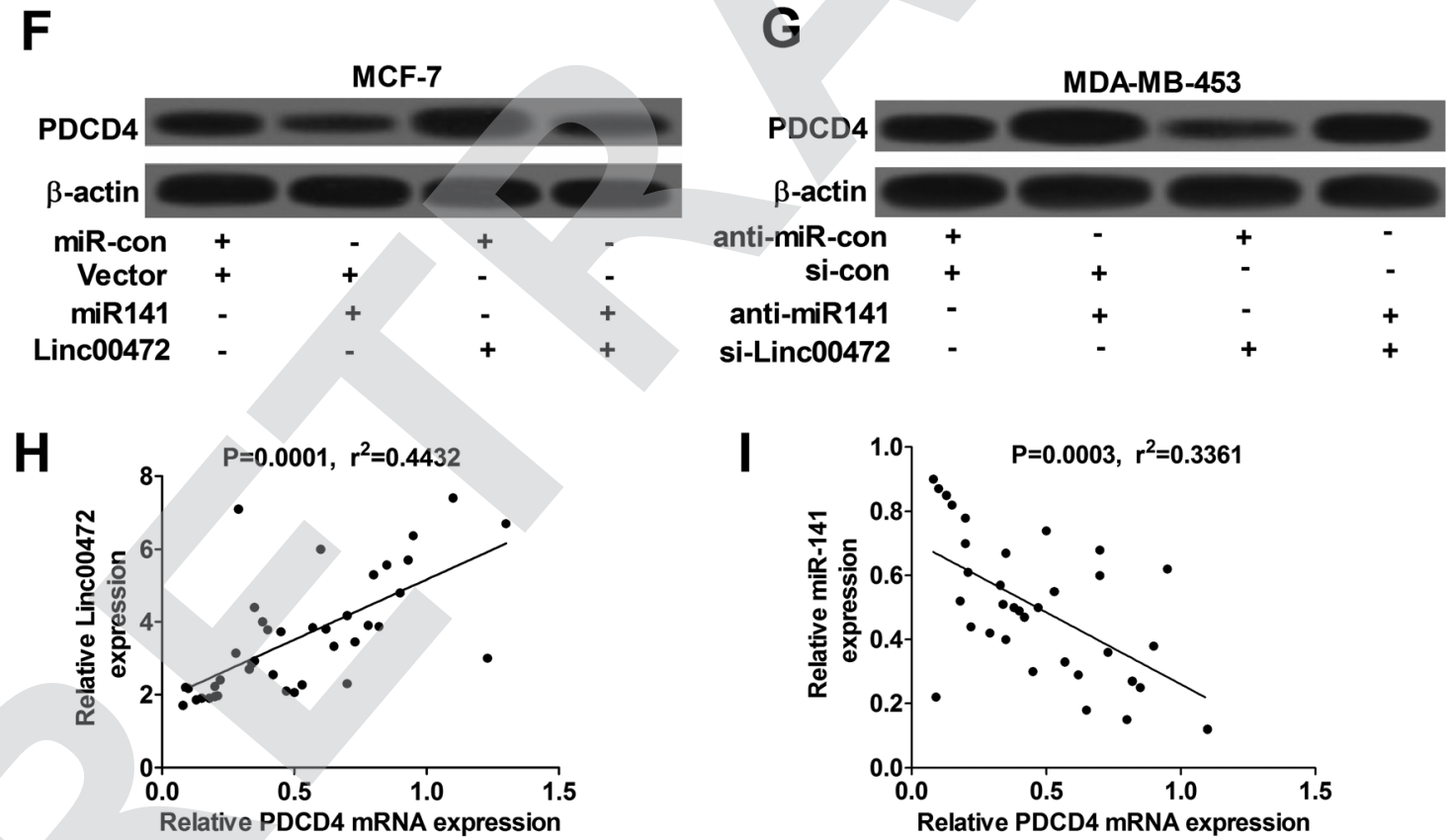

Fig. 5 Linc00472 up-regulated PDCD4 expression by sponging miR-141. (A) The putative binding sites between PDCD4 and miR-141 by TargetScan website and the mutant sites in the mutant-PDCD4 luciferase reporter vector. (B) MCF-7 cells were co-transfected with wide-type or mutant-type PDCD4-3'UTR reporter and miR-con, miR-141, miR-141+pcDNA3.1 vector, or miR-141+pcDNA-linc00472, followed by the detection of luciferase activity $48 \mathrm{~h}$ posttransfection. (C) MDA-MB-453 cells were co-transfected with wide-type or mutant-type PDCD4-3'UTR reporter and anti-miR-con, anti-miR-141, anti-miR-141+si-con, or anti-miR-141+si-linc00472, followed by the analysis of luciferase activity at $48 \mathrm{~h}$ after transfection. (D-G) MCF-7 cells were transfected with miR-con+vector, miR-141+vector, miR-con+linc00472, or miR141+linc00472, and MDA-MB-453 cells were transfected with anti-miR-con+si-con, anti-miR-141+si-con, anti-miR-con+si-linc00472, or antimiR-141+si-linc00472. At $48 \mathrm{~h}$ after transfection, miR-141 expression level was detected by RT-qPCR assays (D and E) and PDCD4 protein level was measured using western blot assays ( $F$ and $G$ ). ( $H$ and I) Spearman's correlation analysis between linc00472 or miR-141 and PDCD4 in 40 cases of breast cancer tumor tissues. $* P<0.05$ 
A

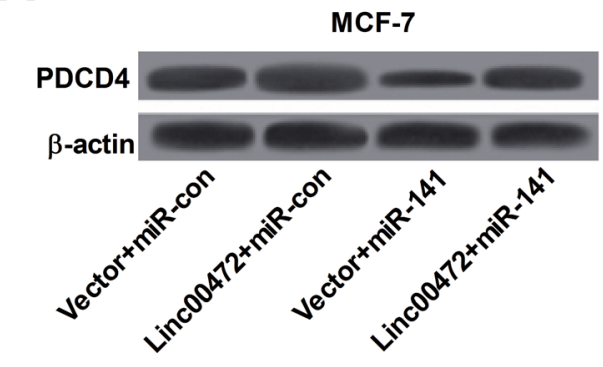

C

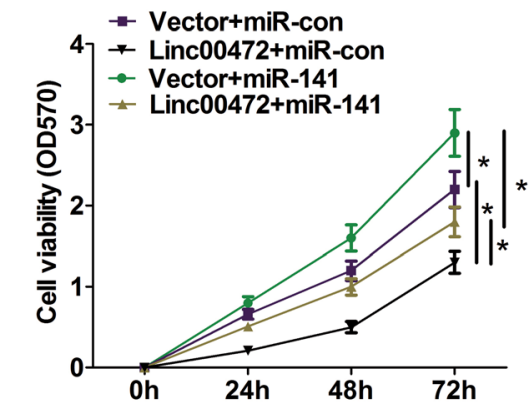

B

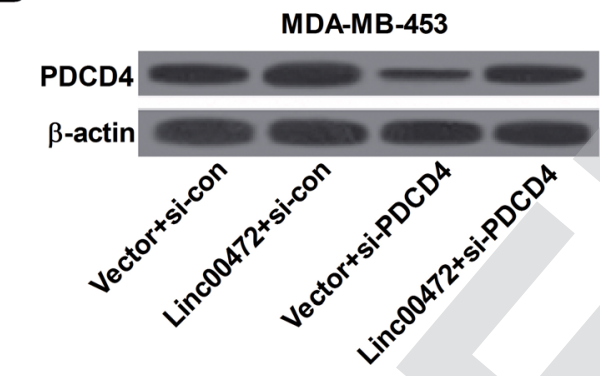

D

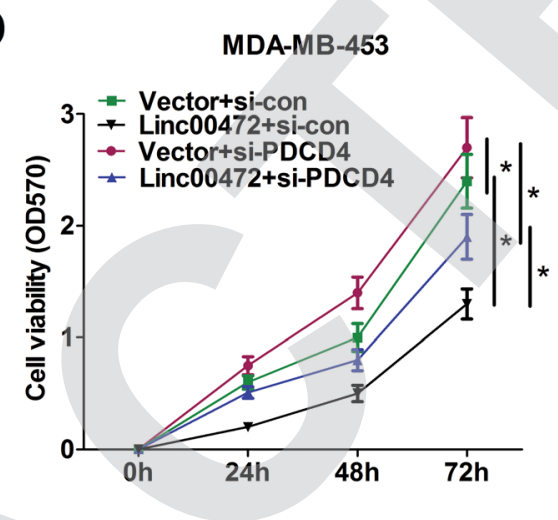

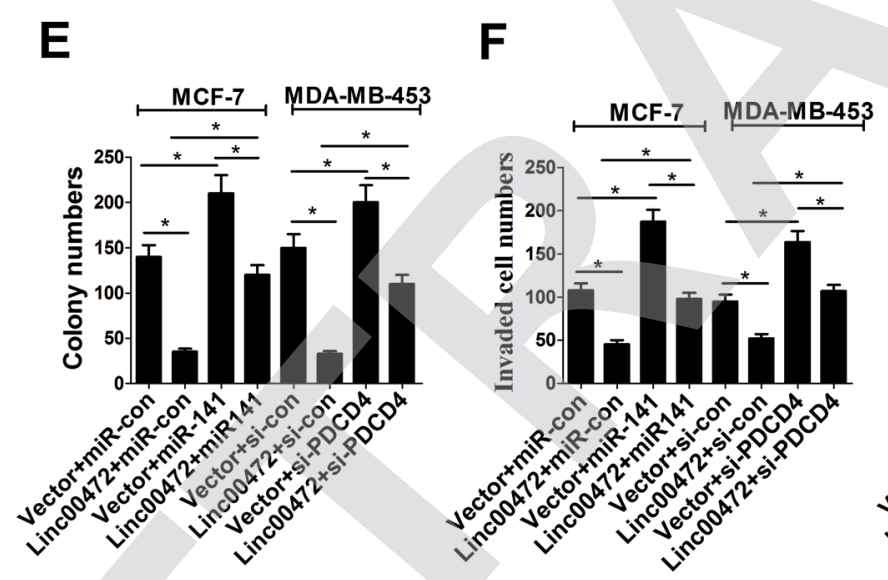

G

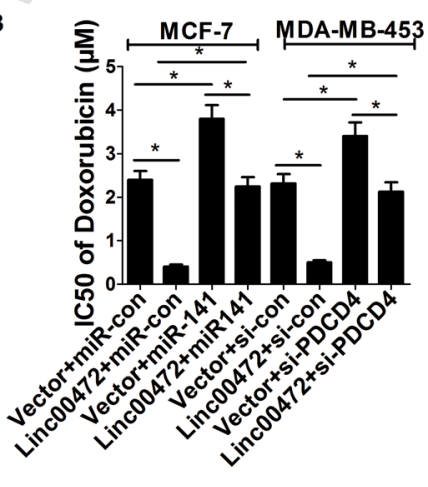

Fig. 6 miR-141 overexpression or PDCD4 knockdown abolished the inhibition effect of linc00472 on breast cancer cell progression. MCF-7 cells were transfected with vector+miR-con, linc00472+miR-con, vector + miR-141 or linc00472+miR-141, and MDA-MB-453 cells were transfected with vector+si-con, linc00472+si-con, vector+si-PDCD4 or linc00472+si-PDCD4, followed by PDCD4 protein levels detection using western blot assays ( $A$ and B), cell proliferation ability assessment by MTT assays ( $C$ and $D)$ and colony formation assays (E), invasion capability evaluation with transwell invasion assays $(F)$, as well as $A D R I C_{50}$ values determination through MTT assays $(G) . * P<0.05$.

the tumor promotion effect of miR-141 was substantially reversed by linc00472 up-regulation. Moreover, enforced expression of linc00472 inhibited tumor growth, whereas linc00472-mediated anti-tumor effect was greatly counteracted following enhanced miR-141 expression. We also compared the expression patterns of miR-141 and linc00472 in linc00472overexpression and control tumor tissues. As expected, transfection of lenti-linc00472 enhanced linc00472 expression and suppressed miR-141 expression (Fig. 7D). Furthermore, miR-141 up-regulation resulted in a decrease of PDCD4 expression and an increase of ki-67 (a proliferation marker) expression level, and linc00472 overexpression resulted in an elevation of PDCD4 expression and a decline of ki-67 expression in resected tumor tissues (Fig. 7E). Moreover, lenti-linc00472-mediated alteration of PDCD4 and ki-67 expression was greatly reversed following the regaining of miR-141 expression (Fig. 7E). These data linc00472 inhibited tumor growth partly through regulating miR-141 and PDCD4 expressions in breast cancer mice models.

\section{Discussion}

LncRNAs and miRNAs have been reported to be implicated in the progression and drug resistance/sensitivity of various cancers including breast cancer. ${ }^{15}$ For instance, downregulation 
A

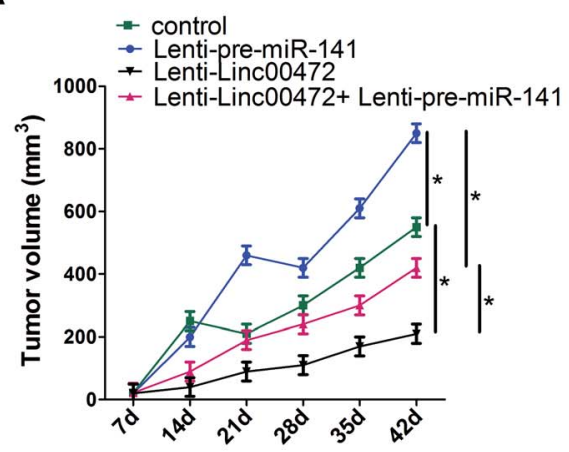

B

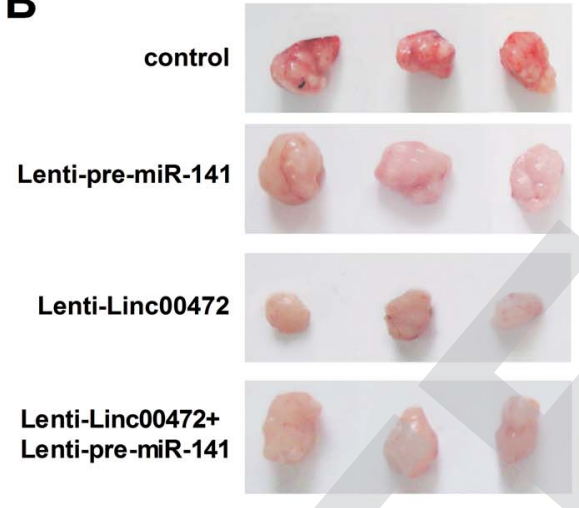

E

E
C

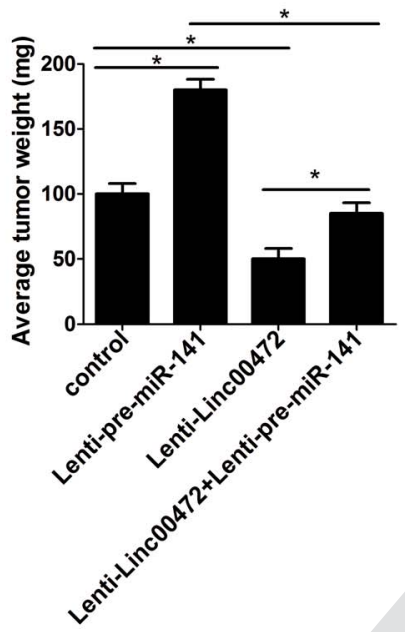

D

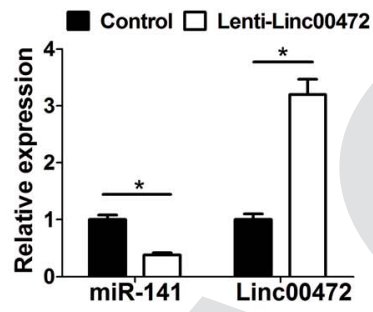

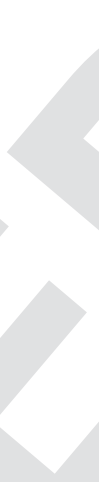
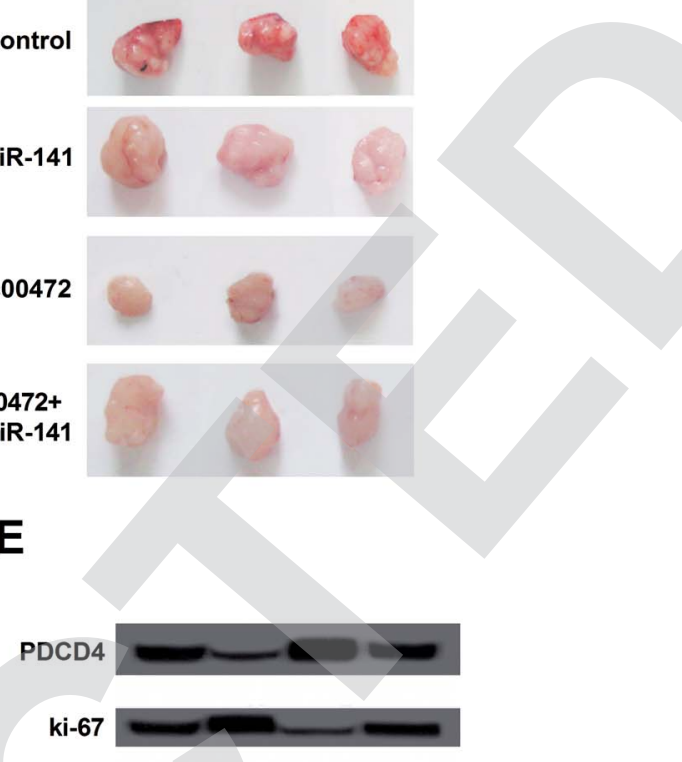

$\beta$-actin

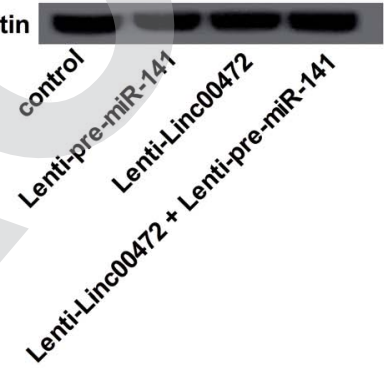

Fig. 7 Linc00472 suppressed tumor growth via modulating miR-141 and PDCD4. MCF-7 cells stably transfected with lenti-con (control), lentipre-miR-141, lenti-linc00472 or lenti-linc $00472+$ lenti-pre-miR-141 were subcutaneously injected into the armpit of nude mice, followed by the detection of tumor volume at 7, 14, 21, 28, 35, 42 days after injection (A), photograph of representative tumors at 42 days after injection (B), measurement of tumor weight at 42 days after inoculation (C), assessment of miR-141 and linc00472 expression levels (D), analysis of PDCD4 and ki-67 protein expression levels (E). $* P<0.05$.

of lncRNA GAS5 enhanced trastuzumab resistance and facilitated proliferation by regulating miR-21/PTEN in breast cancer. ${ }^{16}$ Overexpression of lncRNA BCAR4 promoted proliferation and enhanced endocrine resistance in breast cancer cells. ${ }^{17}$ miR-451 could enhance ADR sensitivity of ADR-resistant breast cancer cells. ${ }^{18}$ Here, our study was performed to elucidate the role and mechanism of linc00472 in breast cancer progression.

In the present study, we firstly demonstrated that linc00472 expression level was down-regulated in breast cancer tissues and cells, and was positively related with favorable disease status and prognosis of breast cancer patients (Fig. 1), which is in accordance with previous studies. ${ }^{13,14}$ Gain-of-function analysis showed that linc00472 overexpression suppressed proliferation and invasion in breast cancer cells (Fig. 2). Moreover, linc00472 overexpression enhanced the susceptibility of breast cancer cells to ADR (Fig. 3), presented by decreased $\mathrm{IC}_{50}$ value of ADR (Fig. 3A and B), increased apoptotic rate (Fig. 3C and D) and enhanced expression of activated caspase 3 (Fig. 3E and F) in breast cancer cells following linc00472 upregulation.
Accumulating evidence indicate that lncRNAs exert functions by acting as ceRNAs of miRNAs, therefore, miRcode website was employed to search for the potential target miRNAs of linc00472. The prediction results showed that miR-141 might interact with linc00472 (Fig. 4A), which was further validated by the following subcellular fractionation (Fig. 4B and C), luciferase (Fig. 4D and E) and RIP (Fig. 4F and G) assays. Furthermore, linc00472 knockdown strikingly promoted miR-141 expression, while linc00472 overexpression induced a notable downregulation of miR-141 level in breast cancer cells (Fig. 4I and J). Additionally, spearman's correlation analysis revealed that miR-141 expression was negatively associated with linc00472 expression in breast cancer tissues (Fig. 4K).

miR-141, a member of miR-200 family, has been validated to be involved in progression and drugs resistance of some cancers by acting as a tumor suppressor or an oncogene. For example, miR-141 expression was down-regulated in gastric cancer and overexpression of miR-141 suppressed the proliferation of gastric cancer cells. ${ }^{19}$ However, some findings revealed that miR-141 was highly expressed in ovarian cancer and high 
expression of miR-141 enhanced cisplatin resistance of ovarian cancer cells. ${ }^{20,21}$ Previous studies also showed that all members of miR-200 family could regulate EGF-driven invasion and miR200a/141 cluster could lead to G1 arrest in breast cancer cells, hinting the association between miR-141 and tumorigenesis of breast cancer. ${ }^{22}$ Moreover, miR-141 expression was found to be strikingly up-regulated in ADR and verapamil resistance breast cancer cell line (MCF-7/AdrVp) compared with parental MCF-7 cell line, indicating a link between miR-141 and ADR resistance of breast cancer cells. ${ }^{23}$ In the present study, we aimed to further explore the role and molecular mechanism of miR-141 in the progression and drug resistance of breast cancer.

Previous studies showed that miRNAs exerted their roles through regulating target mRNAs expression. Hence, TargetScan software was used to figure out the candidate target mRNAs of miR-141 with the results showing that there existed some complementary sites between PDCD4 and miR-141 (Fig. 5A). Then, luciferase (Fig. 5B and C), RT-qPCR (Fig. 5D and E) and western blot (Fig. 5F and $\mathrm{G}$ ) assays further demonstrated that linc00472 performed as a ceRNA of miR-141 to enhance target gene PDCD4 expression. Moreover, spearman's correlation analysis revealed that PDCD4 expression was positively related to linc00472 expression (Fig. 5H), but was inversely associated with miR-141 expression (Fig. 5I).

PDCD4, localized to chromosome 10q24, has been demonstrated to be associated with the development of cancers by regulating various proteins implicated in tumor progression, cell differentiation and cell cycle. ${ }^{\mathbf{2 4 2 5}}$ PDCD4 also has been identified as a tumor suppressor in multiple cancers with a lowlevel expression. ${ }^{2,26}$ For example, overexpression of PDCD4 suppressed ovarian cancer cell proliferation, migration and invasion. ${ }^{27}$ Also, previous studies manifested that PDCD4 inhibited migration and invasion as well as induced apoptosis in breast cancer cells. ${ }^{28,29}$ Moreover, PDCD4 participated in mediating the sensitivity of cancer cells to chemotherapy drugs such as docetaxel, paclitaxel and cisplatin. ${ }^{30,31}$ Taken together, these data promoted us to suppose that linc00472 might affect tumorigenesis and ADR resistance by regulating miR-141 and PDCD4 in breast cancer.

Furthermore, we demonstrated that increased miR-141 or depleted PDCD4 could weaken linc00472-mediated promotive effect on PDCD4 expression in breast cancer cells (Fig. 6A and B). Besides, miR-141 overexpression facilitated proliferation and invasion as well as improved ADR resistance in breast cancer cells (Fig. 6C-G). Restoration assays further unveiled that miR-141 overexpression or PDCD4 knockdown abolished the inhibitory effects of linc 00472 on proliferation, invasion and ADR resistance of breast cancer cells (Fig. 6C-G). Furthermore, we noticed a significant decrease in tumor volume and weight, a notable inhibition of miR-141 and ki-67 expression, as well as a striking increase of PDCD4 expression in linc00472overexpressing mice models. On the contrary, overexpression of miR-141 promoted tumor growth, presented by the increase of tumor volume and weight in mouse xenografts following the upregulation of miR-141. Also, linc00472-mediated anti-tumor effect was partly weakened by miR-141 overexpression and miR-141-induced promotive effect on tumor growth was attenuated following linc00472 up-regulation (Fig. 7A and B). Moreover, PDCD4 expression was decreased in miR-141overexpression tumor tissues, while it was increased in linc00472-overexpression tumor tissues (Fig. 7D and E). All these data indicated that linc00472 suppressed breast cancer progression in vitro and in vivo.

\section{Conclusions}

In a word, our study demonstrated that linc00472 hindered proliferation and invasion, induced apoptosis, and enhanced ADR sensitivity by miR-141/PDCD4 axis in breast cancer cell lines. Our findings contributed to a better understanding of the mechanism of linc00472 involved in breast progression and ADR chemosensitivity, providing a potential biomarker and therapy target for breast cancer.

\section{Author contributions}

Pengwei Lu, Lin Li and Yuanting Gu designed and performed the experiments. Pengwei Lv, Yunqing Yang and Fang Wang analyzed the data and wrote the manuscript. XueYang supervised the study and reviewed the manuscript.

\section{Conflicts of interest}

The authors have no conflict of interest to declare.

\section{Acknowledgements}

Not applicable.

\section{References}

1 R. L. Siegel, K. D. Miller and A. Jemal, Ca-Cancer J. Clin., 2016, 66, 7-30.

2 F. Cardoso, M. Castiglione and E. G. W. Group, Ann. Oncol., 2009, 22, 15-18.

3 T. Gamucci, A. M. D'Ottavio, E. Magnolfi, M. Barduagni, A. Vaccaro, I. Sperduti, L. Moscetti, F. Belli and L. Meliffi, Br. J. Cancer, 2007, 97, 1040-1045.

4 C. E. Desantis, S. A. Fedewa, S. A. Goding, J. L. Kramer, R. A. Smith and A. Jemal, Ca-Cancer J. Clin., 2015, 66, 31-42.

5 G. W. Sledge, D. Neuberg, P. Bernardo, J. N. Ingle, S. Martino, E. K. Rowinsky and W. C. Wood, J. Clin. Oncol., 2003, 21, 588592.

6 C. Marquette and L. Nabell, Curr. Treat. Options Oncol., 2012, 13, 263-275.

7 A. Faversani, V. Vaira, G. P. Moro, D. Tosi, A. Lopergolo, D. C. Schultz, D. Rivadeneira, D. C. Altieri and S. Bosari, Breast Cancer Res., 2014, 16, 1-13.

8 M. Majidinia and B. Yousefi, DNA Repair, 2016, 45, 25-33.

9 Y. Liu, S. Sharma and K. Watabe, Front. Biosci., 2015, 7, 94.

10 X. Li, S. Wang, Z. Li, X. Long, Z. Guo, G. Zhang, J. Zu, Y. Chen and L. Wen, Int. J. Biol. Macromol., 2017, 105, 346-353.

11 X. Xue, Y. A. Yang, A. Zhang, K. W. Fong, J. Kim, B. Song, S. Li, J. C. Zhao and J. Yu, Oncogene, 2016, 35, 2746-2755. 
12 S. J. Shi, L. J. Wang, B. Yu, Y. H. Li, Y. Jin and X. Z. Bai, Oncotarget, 2015, 6, 11652-11663.

13 Y. Shen, D. Katsaros, L. W. Loo, B. Y. Hernandez, C. Chong, E. M. Canuto, N. Biglia, L. Lu, H. Risch and W. M. Chu, Oncotarget, 2015, 6, 8579-8592.

14 Y. Shen, Z. Wang, L. W. Loo, Y. Ni, W. Jia, P. Fei, H. A. Risch, D. Katsaros and H. Yu, Breast Cancer Res. Treat., 2015, 154, 473-482.

15 D. Ayers and J. Vandesompele, Genes, 2017, 8, E95.

16 W. Li, L. Zhai, H. Wang, C. Liu, J. Zhang, W. Chen and Q. Wei, Oncotarget, 2016, 7, 27778-27786.

17 M. Godinho, D. Meijer, B. Setyono-Han, L. C. Dorssers and T. V. Agthoven, J. Cell. Physiol., 2011, 226, 1741-1749.

18 O. Kovalchuk, J. Filkowski, J. Meservy, Y. Ilnytskyy, V. P. Tryndyak, V. F. Chekhun and I. P. Pogribny, Mol. Cancer Ther., 2008, 7, 2152-2159.

19 D. Ying, Y. Xu, D. Ling, H. Yao, Y. Hong, T. Zhou and J. Si, J. Gastroenterol., 2009, 44, 556-561.

20 Y. C. Gao and J. Wu, Tumor Biol., 2015, 36, 4843-4850.

21 M. T. van Jaarsveld, J. Helleman, A. W. Boersma, P. F. van Kuijk, W. F. van Ijcken, E. Despierre, I. Vergote, R. H. Mathijssen, E. M. Berns and J. Verweij, Oncogene, 2013, 32, 4284-4293.
22 S. Uhlmann, J. D. Zhang, A. Schwäger, H. Mannsperger, Y. Riazalhosseini, S. Burmester, A. Ward, U. Korf, S. Wiemann and O. Sahin, Oncogene, 2010, 29, 4297-4306.

23 G. Q. Chen, Z. W. Zhao, H. Y. Zhou, Y. J. Liu and H. J. Yang, Med. Oncol., 2010, 27, 406-415.

24 A. P. Jansen, C. E. Camalier and N. H. Colburn, Cancer Res., 2005, 65, 6034-6041.

25 B. Lankatbuttgereit and R. Göke, Biol. Cell., 2009, 101, 309317.

26 X. Liu, Z. Zhang, L. Sun, N. Chai, S. Tang, J. Jin, H. Hu, Y. Nie, X. Wang and K. Wu, Carcinogenesis, 2011, 32, 1798-1805.

27 N. Wei, S. S. Liu, K. K. Chan and H. Y. Ngan, PLoS One, 2012, 7, e30311.

28 O. Afonja, D. Juste, S. Das, S. Matsuhashi and H. H. Samuels, Oncogene, 2004, 23, 8135-8145.

29 A. N. Santhanam, A. R. Baker, G. Hegamyer, D. A. Kirschmann and N. H. Colburn, Oncogene, 2010, 29, 3921-3932.

30 G. H. Shi, D. W. Ye, X. D. Yao, S. L. Zhang, B. Dai, H. L. Zhang, Y. J. Shen, Y. Zhu, Y. P. Zhu and W. J. Xiao, Acta Pharmacol. Sin., 2010, 31, 867-873.

31 M. Shiota, H. Izumi, A. Tanimoto, M. Takahashi, N. Miyamoto, E. Kashiwagi, A. Kidani, G. Hirano, D. Masubuchi and Y. Fukunaka, Cancer Res., 2009, 69, 3148-3156. 